\title{
MiR-499a-5p promotes 5-FU resistance and the cell proliferation and migration through activating PI3K/Akt signaling by targeting PTEN in pancreatic cancer
}

\author{
Liu Ouyang ${ }^{1 \#}$, Ren-Dong Liu ${ }^{2 \#}$, De-Qiao Lei ${ }^{3 \#}$, Qing-Chao Shang ${ }^{4 \#}$, Hui-Fen Li $^{5}$, Xian-Gui Hu ${ }^{1}$, \\ Hao Zheng ${ }^{6,7}$, Gang Jin ${ }^{1}$
}

${ }^{1}$ Department of General Surgery, Changhai Hospital, Second Military Medical University, Shanghai, China; ${ }^{2}$ Department of Hepatobiliary Surgery, General Hospital of Southern Theatre Command, Guangzhou, China; ${ }^{3}$ Department of General Surgery, General Hospital of Southern Theatre Command, Guangzhou, China; ${ }^{4}$ Department of Radiation Oncology, General Hospital of Southern Theatre Command, Guangzhou, China; ${ }^{5}$ Department of Hepatic Surgery \& Interventional Radiology, Eastern Hepatobiliary Surgery Hospital, Second Military Medical University, Shanghai, China; ${ }^{\circ}$ Department of Reproductive Heredity Center, Changhai Hospital, Second Military Medical University, Shanghai, China; ${ }^{7}$ Third Department of Hepatic Surgery, Eastern Hepatobiliary Surgery Hospital, Second Military Medical University, Shanghai, China

Contributions: (I) Conception and design: G Jin; (II) Administrative support: L Ouyang, RD Liu, DQ Lei, QC Shang; (III) Provision of study materials or patients: HF Li; (IV) Collection and assembly of data: L Ouyang; (V) Data analysis and interpretation: L Ouyang; (VI) Manuscript writing: All authors; (VII) Final approval of manuscript: All authors.

\#These authors contributed equally to this work.

Correspondence to: Gang Jin. Department of General Surgery, Changhai Hospital, Second Military Medical University, Shanghai 200438, China. Email: jingang@smmu.edu.cn; Xian-Gui Hu. Department of General Surgery, Changhai Hospital, Second Military Medical University, Shanghai 200438, China. Email: xianguihu@yahoo.com.cn; Hao Zheng. Department of Reproductive Heredity Center, Changhai Hospital, Second Military Medical University, Shanghai 200438, China. Email: littlestare180710@126.com.

Background: Pancreatic cancer (PC) can be considered a representative cancer type of the human body. As demonstrated by some studies, microRNA (miR)-499 is dysregulated in various cancer types including $\mathrm{PC}$, for which chemotherapy involving 5-fluorouracil (5-FU) has long been considered the first-line therapy. However, there are complex and comprehensive mechanisms related to 5-FU, which have not been fully elucidated. This study thus aimed to examine the molecular mechanisms of 5-FU resistance through miR499a-5p in PC.

Methods: The expression of miR-499a-5p in PC was measured using quantitative polymerase chain reaction (PCR). MiR-499a-5p was examined in-vivo for its effects on the malignant phenotypes of PC cells.

Results: The results of the present study demonstrated miR-499a-5p to be upregulated in PC and 5-FU resistant PC tissues. According to in vitro assays in PC cells (PANC1/FR), miR-499a-5p was found to affect adenosine triphosphate (ATP) binding cassette subfamily B member 1 (P-gp), ATP binding cassette subfamily $\mathrm{C}$ member 1 (MRP1), and ATP binding cassette subfamily G member 2 (BCRP), thereby facilitating 5-FU resistance in PC cells. Functions assays indicated that suppressed miR-499a-5p expression inhibited the proliferation and migration of cells but facilitated apoptosis in the PC cell line; by contrast, miR-499a-5p overexpression triggered the inverse phenotypic changes of cells. Concerning the mechanisms involved, miR-499a-5p increased PI3K/Akt signaling by targeting phosphatase and tensin homolog (PTEN). Conclusions: Taken together, these findings demonstrate that miR-499a-5p can be potentially applied to PC therapy.

Keywords: MiRNA-499a-5p; proliferation; pancreatic cancer (PC); PI3K/Akt; 5-fluorouracil resistance (5-FU resistance)

Submitted Nov 24, 2021. Accepted for publication Dec 22, 2021.

doi: 10.21037/atm-21-6556

View this article at: https://dx.doi.org/10.21037/atm-21-6556 


\section{Introduction}

Pancreatic cancer (PC) can be considered a representative cancer type (1), and the metastasis of PC is the main reason for the death of patients with this disease (2). Individuals with early primary tumors may be effectively treated by surgery or other intervening treatments, which, however, show only limited efficacy for those with advanced cancers $(3,4)$. 5-fluorouracil $(5-\mathrm{FU})$, as a chemotherapeutant that leads to cytotoxic damage, is the basis of standard chemotherapy for PC (5). 5-FU-based chemotherapy, including combined therapy and adjuvant therapy, has seen widespread application in the medical care of different cancer types. Nevertheless, there is obvious variability in the clinical responses to $5-\mathrm{FU}$, and chemoresistance restricts PC therapy to a considerable extent (6). The mechanisms related to 5-FU resistance are complex, and have not been extensively investigated.

MicroRNAs (miRNAs) are small RNA transcripts that do not possess protein-coding capabilities. MiRNAs can bind to the protein-coding transcripts, thereby regulating the development and pathology of animals and humans (7-10). An increasing number of studies have associated miRNAs with different cancer types including PC with regard to their occurrence, progression, and metastasis (11). Studies have pointed to the central importance of miR-499a-5p in various human diseases and biological activities (12-16). Recent studies have shown that miR-499 targets forkhead box $\mathrm{O} 4$ (FOXO4) and programmed cell death 4 (PDCD4), thereby facilitating cell invasion and tumor metastasis in PC (17). However, little is known regarding the relationship between miR-499a-5p and 5-FU resistance in PC. In this study, we focus on the role of miR-499a-5p in promoting 5-FU resistance

The present study found that PC tissues and cells with miR-499a-5p overexpression were resistant to 5-FU-therapy and thatmiR-499a-5p mediated 5-FU resistance through PTEN-PI3K-AKT signaling. Therefore, miR-499a-5p could be a new target of therapy for overcoming PC drug resistance.

We present the following article in accordance with the MDAR reporting checklist (available at https://dx.doi. org/10.21037/atm-21-6556).

\section{Methods}

\section{Tissue samples}

All procedures performed in this study involving human participants were in accordance with the Declaration of Helsinki (as revised in 2013). The present study obtained the approval of the Ethics Committee (No. CHEC-Y2020-043) of Changhai Hospital (Shanghai, China) and written informed consent was obtained from all participants. We collected 45 pairs of PC tissues together with corresponding normal adjacent tissues from PC patients who received surgical resection at the Changhai Hospital from January 2011 to December 2015. All of them were from patients who received initial surgery and did not accept preoperative chemotherapy or radiotherapy. The tissue samples were immediately frozen in the liquid nitrogen during surgery and stored at $-80{ }^{\circ} \mathrm{C}$ for later use. Table 1 lists the patients' clinicopathological features.

\section{Cell culture, transfection and reagent}

Human embryonic kidney (HEK) 293 T cells, the human PC cell lines (Mia-capa2, PANC1, AsPC-1, and Capan-2), and the pancreas cell line HPDE6-C7 were acquired from the Chinese Academy of Sciences (Shanghai, China). PANC1/ FU PC cancer cell line (5-FU-resistant) were purchased from Mei Xuan Biological Science and Technology Ltd. (Shanghai, China). All cells received culturing in Dulbecco's Modified Eagle Medium (DMEM; Gibco, Grand Island, NY, USA) that contained fetal bovine serum (FBS; $10 \%$, Gibco) added with penicillin-streptomycin (1\%, SigmaAldrich, St-Louis, MO, USA) in a humid incubator with 5\% $\mathrm{CO}_{2}$ at $37{ }^{\circ} \mathrm{C}$. The mimics control, miR-499a-5p mimics, inhibitor control, and miR-499a-5p inhibitors were acquired from Gene Copoeia (Guangzhou, China). Lipofectamine 2000 reagent (Invitrogen, Thermo Fisher Scientific, and Waltham, MA, USA) was applied to cell transfection in line with the manufacturer's instructions. PC cells underwent 12 hours of treatment with $1 \mu M$ of MK2206, an Akt inhibitor (Selleck Chemicals, Houston, TX, USA).

\section{Real-time quantitative polymerase chain reaction (qRT- PCR)}

An RNA extraction kit (Co Win Bioscience Co., Ltd., Beijing, China) assisted in extracting the total RNA and miRNA cDNA Synthesis Kit (Co Win Bioscience Co., Ltd.) assisted in converting the extracted total RNA to complement DNA (cDNA). A miRNA qPCR detection kit (Co Win Bioscience Co., Ltd.) helped to examine the miRNA level. The thermocycling conditions were as follows: 5 minutes at $95{ }^{\circ} \mathrm{C}, 40$ cycles at $95^{\circ} \mathrm{C}$ for $10 \mathrm{~s}$, 
Table 1 Association between miR-499a-5p expression and clinicopathologic characteristics of PC patients in the study cohort

\begin{tabular}{|c|c|c|c|}
\hline Characteristics & \multicolumn{2}{|c|}{ No. of patients } & $P$ value \\
\hline Gender & & & 0.903 \\
\hline Female & 9 & 9 & \\
\hline Male & 14 & 13 & \\
\hline$<60$ & 5 & 7 & \\
\hline$\geq 60$ & 18 & 15 & \\
\hline Tumor location & & & 0.626 \\
\hline Rectum & 10 & 8 & \\
\hline Well + moderate & 16 & 16 & \\
\hline Poor & 7 & 6 & \\
\hline Tumor size $(\mathrm{cm})$ & & & $0.047^{\star}$ \\
\hline$<5$ & 13 & 6 & \\
\hline$\geq 5$ & 10 & 16 & \\
\hline Local invasion & & & 0.372 \\
\hline pT1-T2 & 15 & 17 & \\
\hline рT3-pT4 & 8 & 5 & \\
\hline III & 9 & 18 & \\
\hline
\end{tabular}

${ }^{\mathrm{a}}$, Pearson chi-square test was used for comparison between subgroups; *, statistical significance.

$60{ }^{\circ} \mathrm{C}$ for $30 \mathrm{~s}$, and $72{ }^{\circ} \mathrm{C}$ for $1 \mathrm{~s}$. Each reaction was repeated 3 times. The $2^{-\Delta \Delta \mathrm{Ct}}$ method was used to calculate miR-2053 expression (18). The relative gene expression was analyzed by 3 independent experiments.

\section{Cell viability assays}

The cell viability assays were conducted according to the methods outlined by Du et al. (19). Transfected PANC1 and PANC1/FR cells underwent 24 hours of treatment by $5-\mathrm{FU}(0-20 \mu \mathrm{M})$ for their cell viability to be assessed by
Cell Counting Kit-8 (CCK-8) assays. A microscope was employed for reading the absorbances at $450 \mathrm{~nm}$. After the normalization of values to the untreated controls, we calculated the half maximal inhibitory concentration (IC50s) for each treatment.

\section{Bromodeoxyuridine proliferation}

The indicated PC cell lines were treated by using miR$499 \mathrm{a}-5 \mathrm{p}$ mimics or inhibitors or the corresponding controls. After 10 hours, we used trypsin to digest the cells and 
seeded 10,000-20,000 cells into 96-well plates. BrdU Cell Proliferation Assay Kit (\#6813, Cell Signaling Technology, Danvers, MA, USA) was then applied.

\section{Caspase-3/7 assay}

The caspase-3/7 activity following the indicated PC cell lines were treated by using miR-499a-5p mimics or inhibitors or the corresponding controls, and detected by the Caspase-Glo 3/7 Assay kit (G8090, Promega, Madison, WI, USA).

\section{Western blotting}

RIPA buffer was used to extract the proteins from PC cells, and a bicinchoninic acid (BCA, Promega, Madison, WI, USA) kit assisted in measuring the concentration. Subsequently, proteins underwent electrophoresis with $10 \%$ SDS-PAGE and were transferred onto polyvinylidene fluoride (PVDF) membranes (Bio-Rad, Hercules, CA, USA). The membranes were then plated in $5 \%$ nonfat milk for blockage and incubated with the following primary antibodies at $4{ }^{\circ} \mathrm{C}$ : PTEN $(1: 1,000$, \#9188; Cell Signaling Technology), Akt (1:1,000, \#4691; Cell Signaling Technology), anti-N-Cadherin (1:1,000, \#ab18203, Abcam, Cambridge, UK), p-Akt (1:2,000, \#4060; Cell Signaling Technology), anti-E-Cadherin (1:1,000, \#ab1416, Abcam), anti-Vimentin (1:1,000, \#ab92547, Abcam), and GAPDH (1:1,000, \#5174; Cell Signaling Technology). Following this, the membranes were incubated for $1 \mathrm{~h}$ with the second antibody (anti-rabbit \#7074; Cell Signaling Technology) at room temperature. At last, the membranes were detected by an enhanced chemiluminescence (ECL) reagent (Beyotime Institute of Biotechnology, Haimen, China).

\section{Luciferase reporter assay}

We inserted the synthesized wild-type (WT) or mutant (MT) 3'UTR of PTEN messenger RNA (mRNA) downstream of the pEZX-MT06 vector (Gene Copoeia), using wt-3'UTR and mut-3'UTR of PTEN to transfect 293T cells that had been transfected by miR-499a-5p mimics or controls, respectively. Cell collection was completed 48 hours later. LucPair Duo- Luciferase Assay Kit (Gene Copoeia) was used to quantify the luciferase activity.

\section{Statistical analysis}

GraphPad Prism 7.0 software (GraphPad Software, Inc.,
San Diego, CA, USA) assisted in analyzing data, which are expressed as the mean \pm SD. The paired Student's $t$ test was carried out for determining and analyzing the statistical significance regarding the difference between PC tissues and normal samples. One-way analysis of variance (ANOVA) was used to confirm the significant differences between multiple tests. Student-Newman-Keuls test acted as the post hoc test after the ANOVA. The $\chi^{2}$ test was used for analyzing the degree to which miR-499a-5p expression affected the clinicopathological characteristics. All the experiments were repeated 3 times. $\mathrm{P}$ values $<0.05$ were considered for statistical significance.

\section{Results}

miR-499a-5p was overexpressed in PC tissue and cell lines

Firstly, qRT-PCR was used to assess miR-499a-5p expression in 45 paired PC samples. MiR-499a-5p showed a higher expression in $\mathrm{PC}$ versus the adjacent noncancerous tissue (Figure $1 A$ ). In patients resistant to $5-\mathrm{FU}$, miR-499a$5 \mathrm{p}$ was upregulated vs. 5-FU-sensitive patients (Figure $1 B$ ). We measured the miR-499a-5p level in various PC cell lines and compared them with that of the pancreas cell line, HPDE6-C7. In a manner similar to that observed in tissue samples, all PC cells lines examined, including Miacapa-2, PANC1, AsPC-1, and Capan-2, exhibited a higher expression of miR-499a-5p than did normal HPDE6-C7 cells $(\mathrm{P}<0.05$ vs. each $\mathrm{PC}$ cell line; Figure 1C). 5-FU-resistant cells (PANC1/FR) also showed higher miR-499a-5p expression than didPANC1 cells (Figure 1D). To determine how miR-499a-5p expression affects the clinicopathologic characteristics, 45 patients included in the study cohort were placed into 2 subgroups, including a high expression group and a low expression group, and the median expression in PC tissues was considered as the cutoff. Increased miR499a-5p levels in PC led were associated with tumor size $(\mathrm{P}=0.047)$, an increase in lymph node metastasis $(\mathrm{P}=0.048)$, and advanced TNM stage $(\mathrm{P}=0.003$; Table 1$)$. Considered cumulatively, these findings suggest that increased miR499a-5p expression may lead to malignant PC progression.

\section{MiR-499a-5p enhanced 5-FU resistance in PC cells}

We found that in PC cells (PANC1), adose5-FUled to miR499a-5p upregulation (Figure 2A). MiR-499a-5p expression was overexpressed or silenced exogenously to evaluate its significance for PANC1/FR cells (Figure 2B,2C). 5-FU 
A

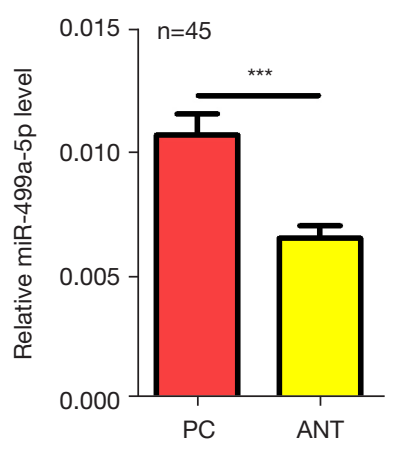

C

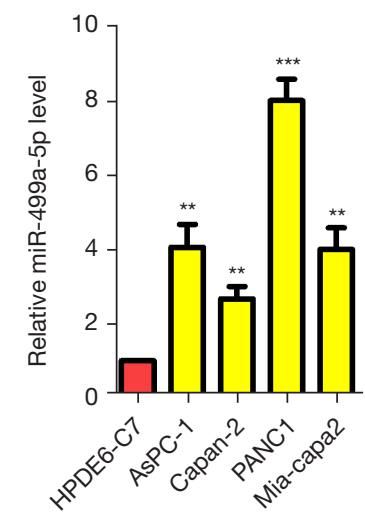

B

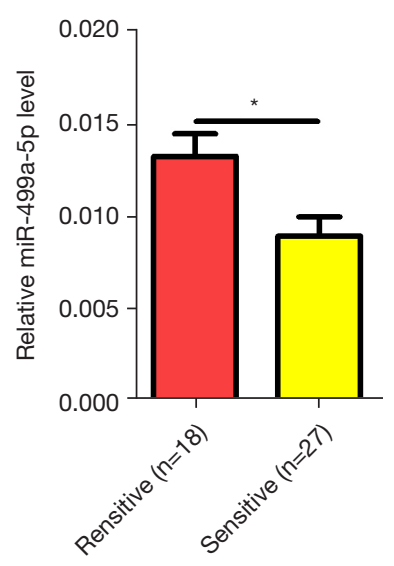

D

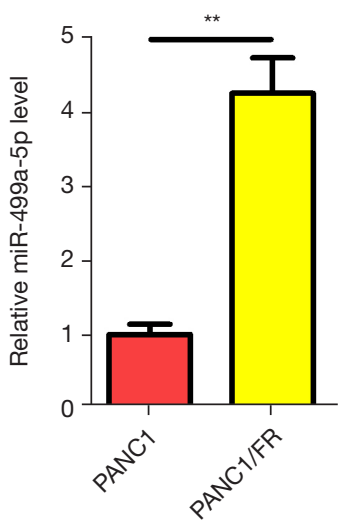

Figure 1 MiR-499a-5p was overexpressed in PC tissue and cell lines. (A) MiR-499a-5p under the assessment of qRT-PCR analysis involving 45 paired PC tissues. (B) MiR-499a-5p level in PC patient with 5-FU resistance $v s$. those with 5-FU sensitivity. (C) MiR-499a-5p level in PC cells vs. control cells under qRT-PCR. (D) MiR-499a-5p expression in PANC1/FR (5-FU resistant) and naive PANC1 cells. *, P<0.05; **, $\mathrm{P}<0.01$; ${ }^{* *}, \mathrm{P}<0.001$. PC, pancreatic cancer; qRT-PCR, real-time quantitative polymerase chain reaction.

was associated with increasedIC50 in PANC1 cells with exogenous miR-499a-5p expression, inhibited miR-499a-5p expression, and showed decreased IC50 in PANC1/FR cells (Figure 2D). As revealed by qRT-PCR analysis, miR-499a$5 \mathrm{p}$ overexpression raised the P-gp, MRP1, and BCRP levels in PANC1, which, however, were suppressed due to miR499a-5p silencing in PANC1/FR (Figure 2E,2F). According to these findings, miR-499a-5p enhanced 5-FU resistance in PC cells.

\section{The oncogenic properties of miR-499a-5p in PC}

For investigating the biological functions of miR-499a$5 \mathrm{p}$ regarding $\mathrm{PC}$ cell progression in vitro, $\mathrm{PC}$ cell lines with miR-499a-5p overexpression with miR-499a$5 \mathrm{p}$ mimics in PANC1 and Mia-capa2 cells (Figure $3 A$ ). Results of BrdU incorporation showed that miR-499a-5p overexpression remarkably improved PANC1 and Miacapa2 cell proliferation (Figure 3B). Moreover, miR-499a-5p overexpression in PANC1 and Mia-capa2 cells significantly increased migration of indicated PC cells (Figure 3C). Moreover, miR-499a-5p overexpression in PANC1 and Mia-capa2 cells significantly inactivated caspase-3-and caspase-7-positive apoptotic cells, respectively, relative to controls (Figure 3D). Meanwhile, miR-499a-5p knockdown in PANC1 and Mia-capa2 cells with miR-499a-5p inhibitor (Figure $3 E$ ) in PANC1 and Mia-capa2 cells inhibited cell proliferation (Figure $3 F$ ), migration ability (Figure $3 G$ ), and 
A

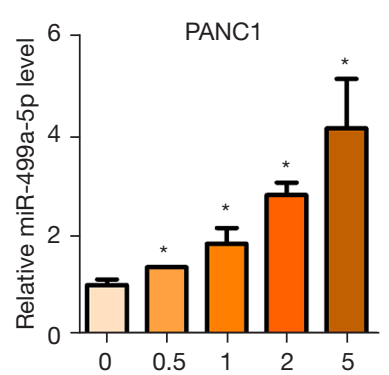

D

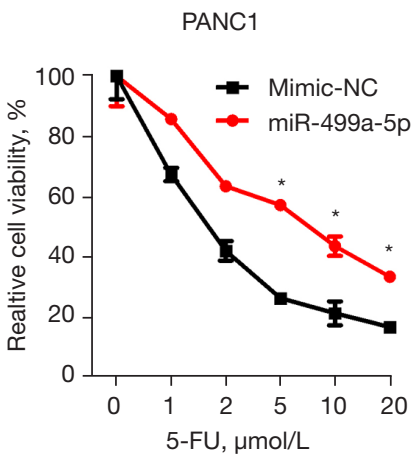

$\mathrm{E}$

PANC1
B

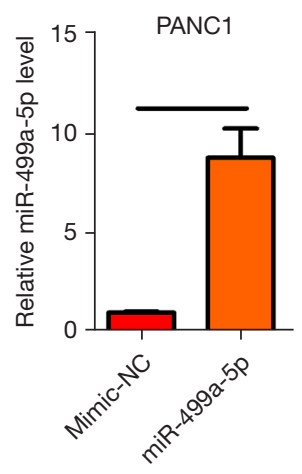

C
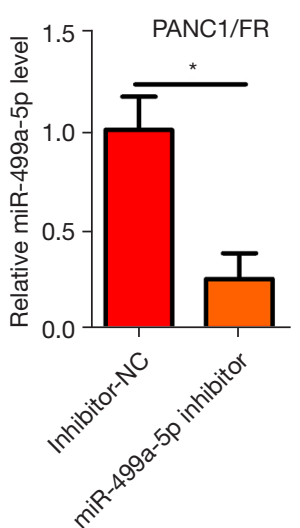
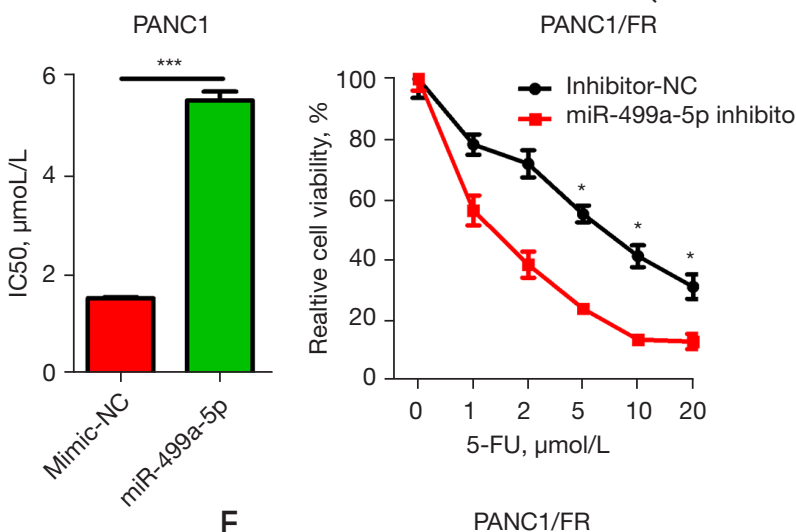

$\mathrm{F}$
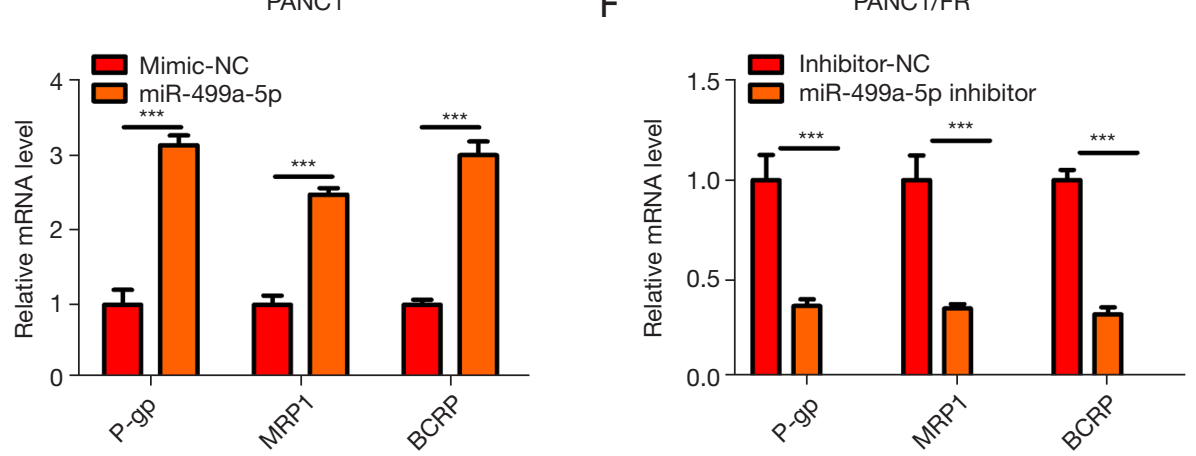

Figure 2 MiR-499a-5p enhanced 5-FU resistance in PC cells. (A) qRT-PCR showing MiR-499a-5p expression in PANC1 cells treated with 5-FU. (B,C) PANC1 cells over-expressing miR-499a-5p or silenced for miR-499a-5p in PANC1/FR. (D) Assessment of cell viability and IC50 of 5-FU in PANC1 vs. PANC1/FR cells. (E,F) P-gp, MRP1, and BCRP expression were assessed by qRT-PCR. *, P<0.05; ***, $\mathrm{P}<0.001$. PC, pancreatic cancer; qRT-PCR, real-time quantitative polymerase chain reaction

activated caspase-3- and caspase-7-positive apoptotic cells, respectively, relative to controls (Figure $3 H$ ). Above results indicated that miR-499a-5p is an oncogene of PC.

\section{MiR-499a-5p enbanced 5-FU resistance and PC metastasis through PI3K-AKT silencing}

For exploring the mechanisms by which miR-499a$5 \mathrm{p}$ enhances 5 -FU resistance and acts as oncogenic driver of PC, the function of miR-499a-5p in epithelialmesenchymal transition (EMT), a vital cancer metastasis pathway, was first identified. MiR-499a-5p notably affected EMT through Vimentin, and N-cadherin essentially maintained the original expression between miR-499a-5p overexpressing or silenced lines (Figure $4 A, 4 B$ ), suggesting that metastasis and drug resistance mediated by miR-499a5pin PC cells were independent of EMT. TargetScan (http:// www.targetscan.org/vert_72/), a public database, was further 

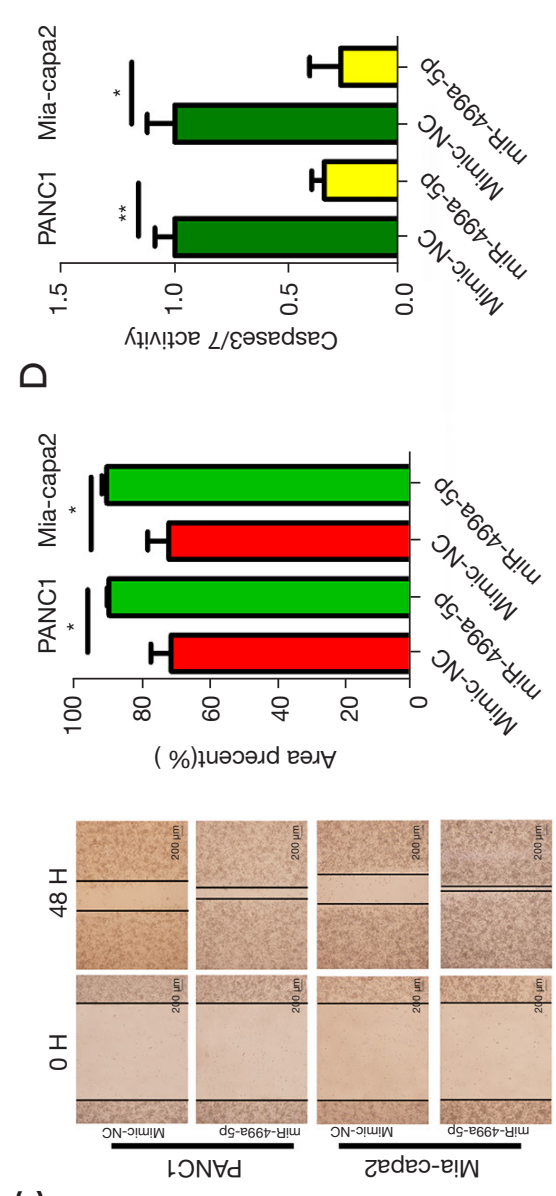

U

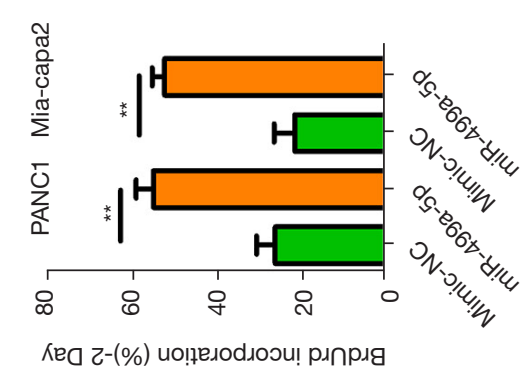

$\infty$

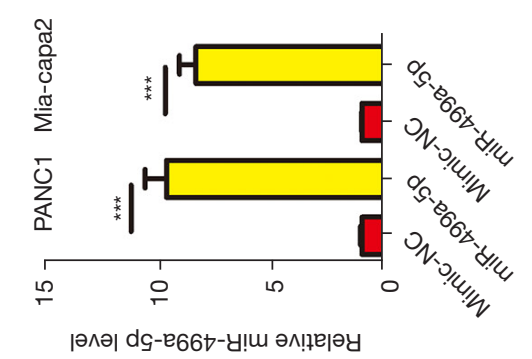

$\varangle$

ш

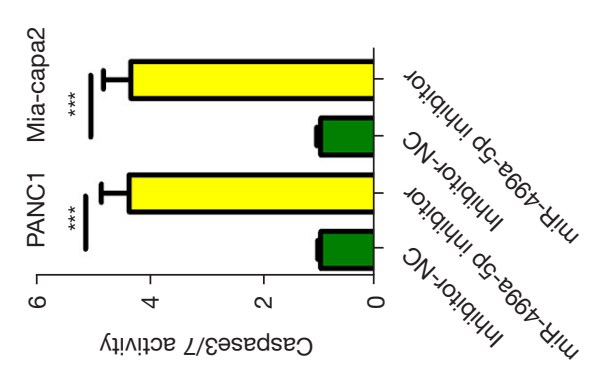

I

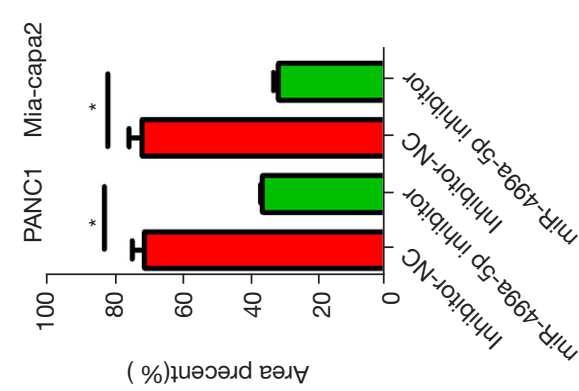

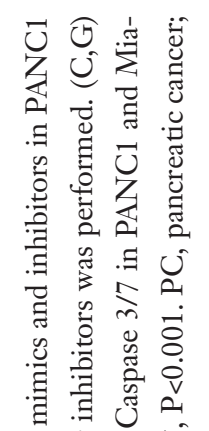

in के

紊.

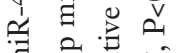

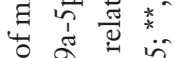

仓ั

远解

पै
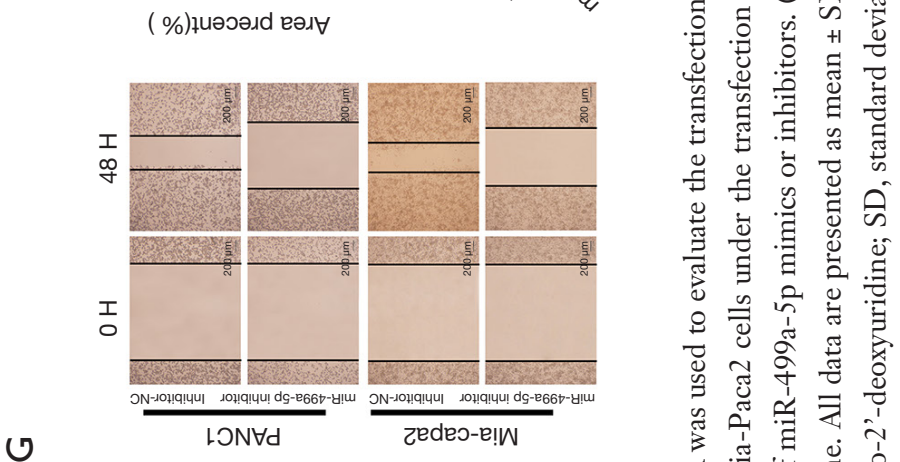

$\checkmark$

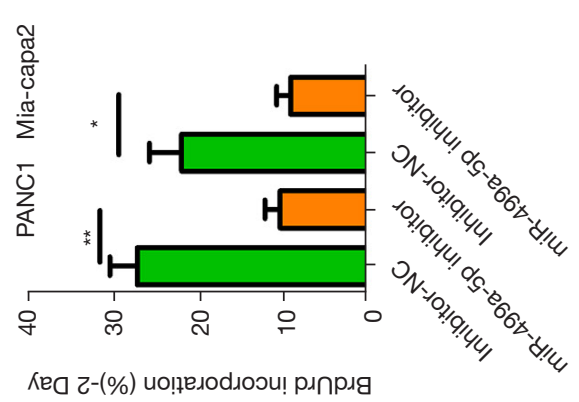

レ

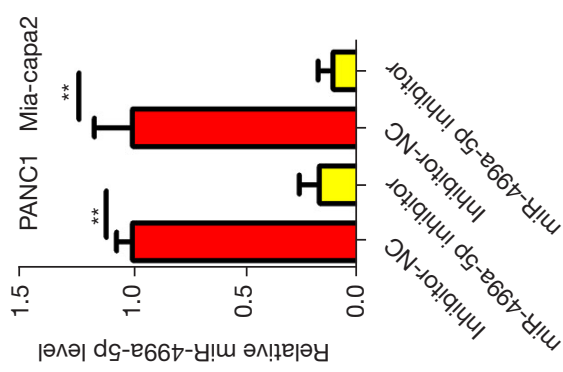

ป ป

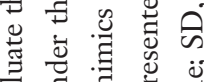

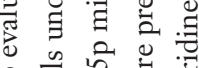

० च की

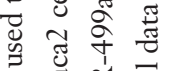

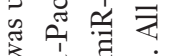

孚芦

Ч

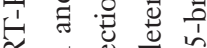

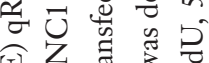

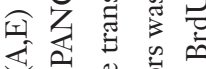

ن $\begin{gathered}0 \\ 0\end{gathered}$

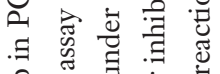

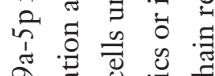

文跑

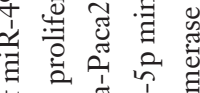

范

○

. ڤै चु

ठัँ

元品

ข

के पु

苟离

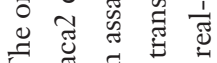

E

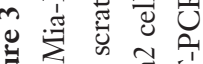

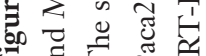


A

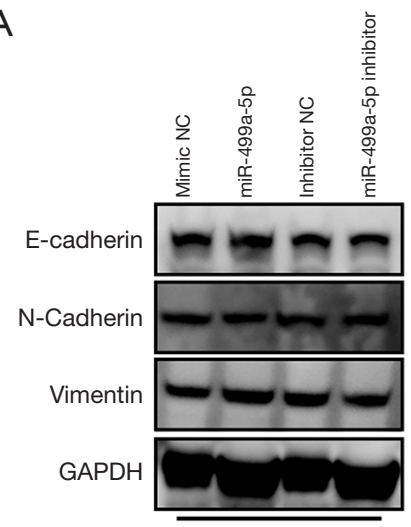

PANC1

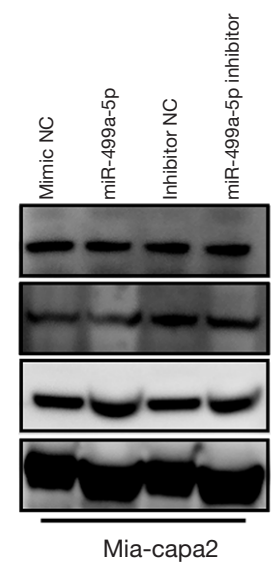

B
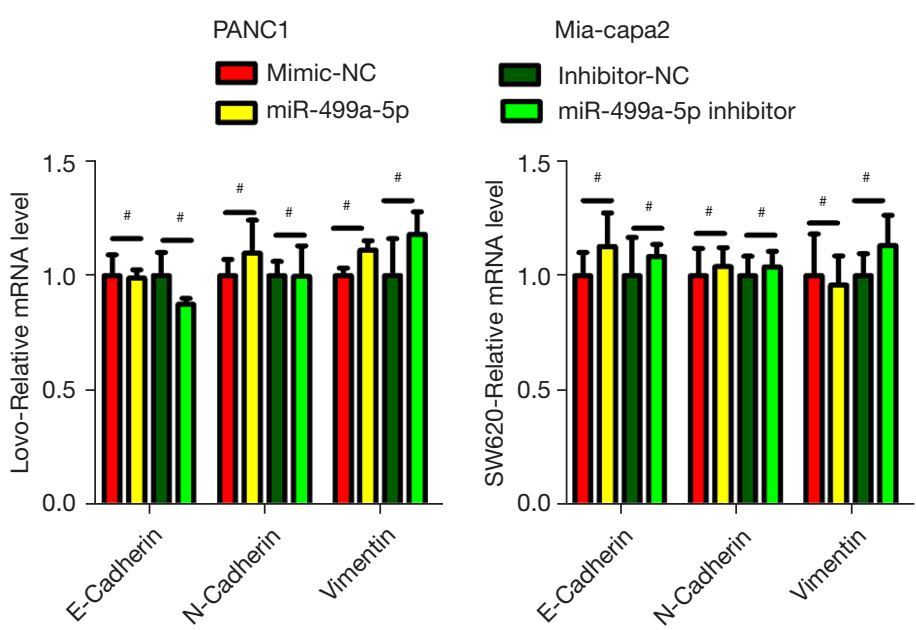

C

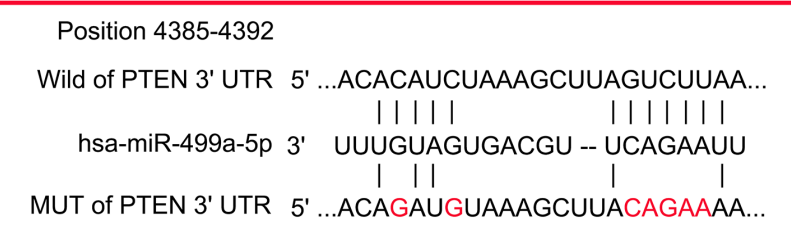

D

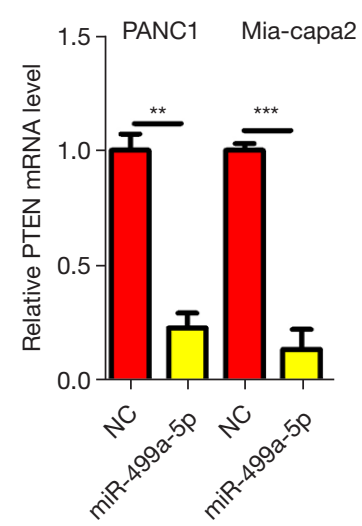

$\mathrm{E}$

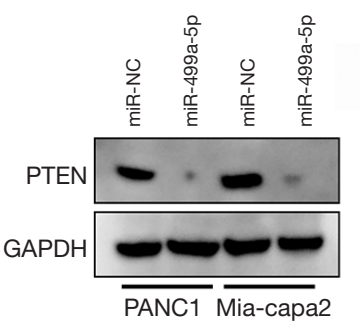

$\mathrm{F}$

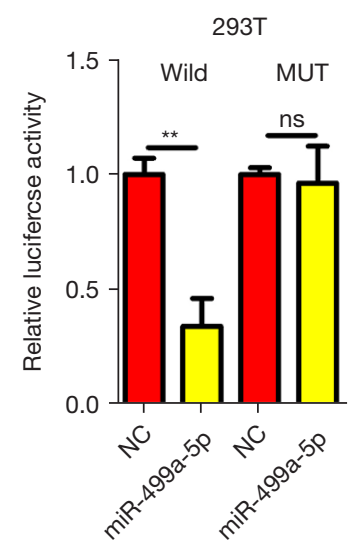

Figure 4 PTEN was a downstream target of miR-499a-5p. (A,B) The EMT (e-cadherin, n-cadherin, and vimentin) markers of mRNA and protein subjected to miR-499a-5p overexpression or underepxression in PANC1 and Mia-Paca2 cells did not change. (C) Putative binding sites for miR-499a-5p in the PTEN mRNA overexpression or underexpression groups, with the mutated sites being underlined. (D,E) miR499a-5p overexpression led to a decrease in PTEN at the mRNA and protein level in PANC1 and Mia-Paca2 cells. N=3. (F) miR-499a$5 \mathrm{p}$ level modulation in 293 $\mathrm{T}$ cells exerted a negative regulatory effect on the luciferase activity exhibited by vectors that contained wt 3'UTR of PTEN relative to vectors that contained MT 3'-UTR. All experiments were repeated 3 times. Data are presented as mean \pm SD. ", $\mathrm{P}>0.05 ;{ }^{* *}, \mathrm{P}<0.01$; ${ }^{* * *}, \mathrm{P}<0.001$. PTEN, phosphatase and tensin homolog deleted on chromosome ten; EMT, Epithelial-Mesenchymal Transition; WT, wild type; MT, mutant type; UTR, untranslated region; SD, standard deviation.

used to search for candidate targets genes specific to miR$499 \mathrm{a}-5 \mathrm{p}$. Based on the query conducted, PTEN possessed a complementary sequence of the miR-499a-5p (Figure 4C). To determine whether miR-499a-5p affects PTEN expression in PC, PTEN expression in the indicated PC cells under the transfection of miR-499a-5p mimics was measured. The data demonstrated that transfection with miR-499a-5p mimics significantly decreased the expression 
A

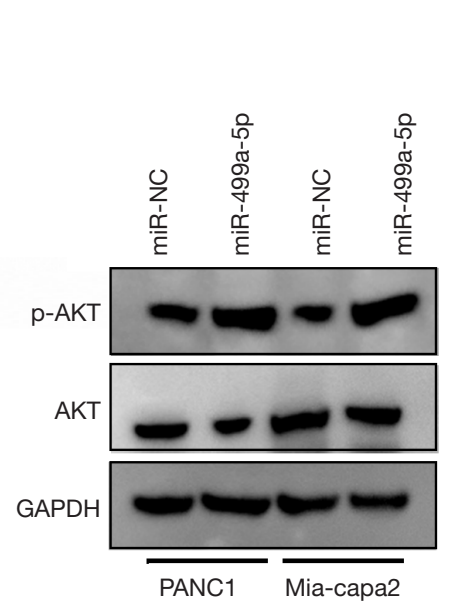

C

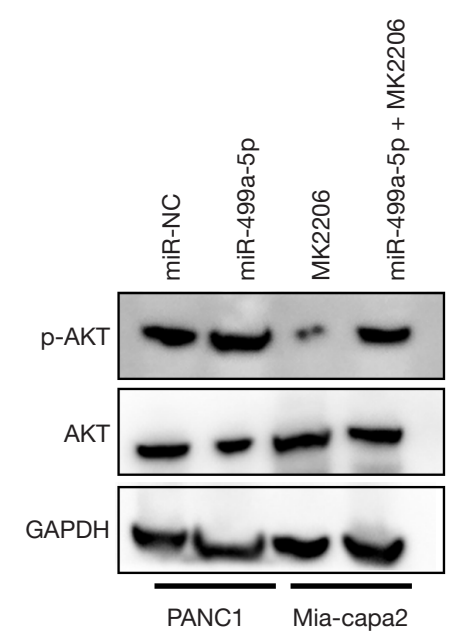

B
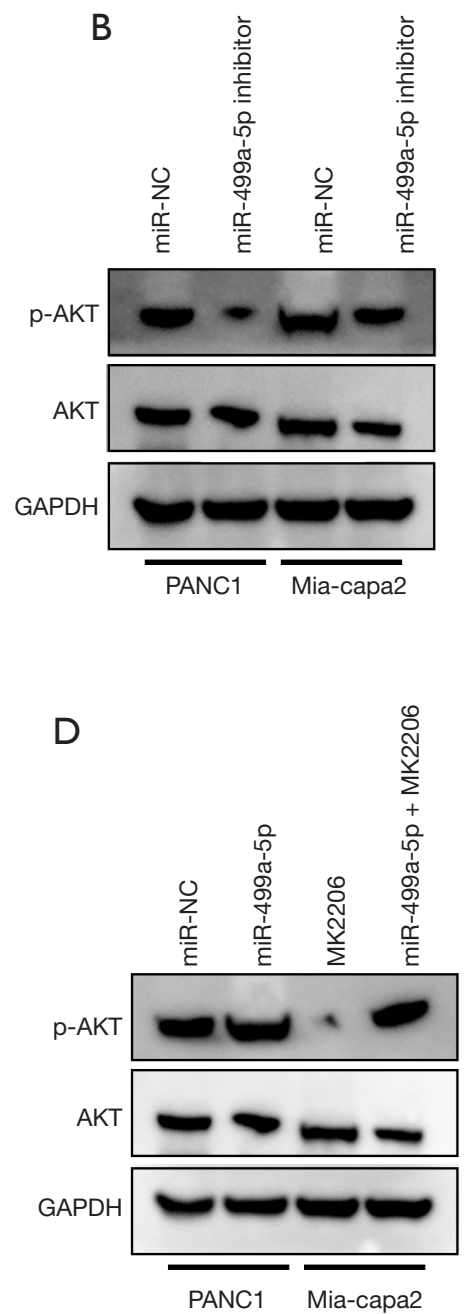

Figure 5 PI3K/Akt pathway was essential for the biological function of miR-499a-5p in PC. (A,B) Western blot analysis demonstrated the promoting and suppressing effect of miR-499a-5p mimics on p-Akt expression in PANC1 and Mia-Paca2 cells, respectively. (C,D) The p-Akt, Akt levels in PANC1 and Mia-Paca2 cells with overexpressed miR-499a-5p under the treatment of MK2206 (an Akt inhibitor) and DMSO. All experiments were repeated 3 times. PC, pancreatic cancer; DMSO, dimethyl sulfoxide.

levels of PTEN mRNA and protein relative to the negative control (NC) group $(\mathrm{P}<0.01$; Figure $4 D, 4 E)$. As revealed by the luciferase reporter assay, $293 \mathrm{~T}$ cells under the transfection of wt PTEN 3'-UTR exhibited decreased luciferase activity as miR-499a-5p levels were restored $(\mathrm{P}<0.05$ for both cell lines; Figure $4 F)$. With regard to the control experiment, miR-499a-5p level modification did not affect the luciferase activity in PANC1 or Mia-capa2 cells under the transfection of mt PTEN 3'-UTR (Figure4F). These findings thus suggest that PTEN expression in PC is directly controlled by miR-499a-5p.

\section{The essential role of PI3K/Akt pathway in the biological function of miR-499a-5p in PC}

As it appeared that PTEN was primarily responsible for negatively regulating PI3K/Akt pathway, we further examined whether miR-499a-5p is capable of changing the activity exhibited by PI3K/Akt pathway in PC cells. As revealed by the western blotting, miR-499a-5p mimics and inhibitors increased and decreased the expression of p-Akt inPANC1 and Mia-Paca2, respectively (Figure $5 A, 5 B$ ). To determine if PI3K/Akt pathway was capable of mediating the degree to which miR-449a-5p affected PC cell 

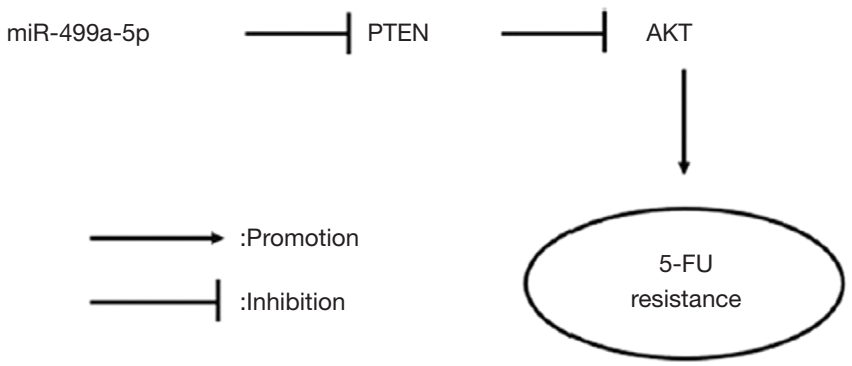

Figure 6 The proposed model of miR-499a-5p attenuating the sensitivity of PC cancer cells to 5-FU. PC, pancreatic cancer; PTEN, phosphatase and tensin homolog deleted on chromosome ten.

progression, MK2206 (an Akt inhibitor) assisted in blocking the activation of Akt in PANC1 and Mia-Paca2 cells with overexpressed miR-499a-5p (Figure 5C,5D).

\section{Discussion}

High rates of 5-FU resistance lead treatment of PC greatly discounted (20). Previous studies have reported that miRNAs have an impact on multidrug resistance (21-23). However, the link between miR-499a-5p and 5-FU resistance has not been reported for PC. The current study indicated that miR-499a-5p was upregulated in PC tissues and cells that showed resistance to 5-FU-therapy and that miR-499a-5p downregulation increased the sensitivity of PANC1/FU to 5-FU. Conversely, miR-499a5 poverexpression in PANC1 cells elevated its IC50 to 5-FU. Further cell line evidence supported miR-499a-5p's function in drug resistance in PC. Previous studies indicated that miRNA-2 1 induces 5-FU resistance by PTEN and PDCD4, the innovation of our study was found that the novel targeted genes (such as P-gp, MRP1, and BCRP) of miR-499a-5p regulated in PC.

PTEN is a type of tumor suppressor gene that has powerful functions and is closely related to apoptosis (24). Several studies have shown that various miRNAs can regulate PTEN in human cancers, including miR-19a in leukemia (25), miR-22 in prostate cancer (26), miR26a in high-grade glioma (27), and miR-21 in HCC (28). Nevertheless, few studies have investigated the association of PTEN with miR-499a-5p in PC. Our study conclusively showed that PTEN is a direct function target for miR$499 a-5 p$. First, it was found that miR-499a-5p had a negative modulating impact on the expression of PTEN in PC cells at the protein and mRNA levels. It was further demonstrated that miR-499a-5p could impact the luciferase activity possessed by wt-3'UTR of PTEN relative to the mut-3'UTR. These findings point to PTEN as a direct function target for miR-499a-5p and suggest that it is capable of mediating the function of miR-499a-5p in PC cells. Decreased PTEN expression can activate the PI3K/ AKT pathway to enhance tumor progression (29-32). Activated Akt is involved in regulating cell cycle and the proliferative, antiapoptotic, metastatic, and invasive abilities of cancer cells (33). The AKT phosphorylation level decreased markedly due to miR-499a-5p downregulation while p-AKT was highly expressed due to miR-499a$5 \mathrm{p}$ upregulation, but AKT expression did not in the 2 conditions.

In summary, our study showed that miR-499a-5p makes PC cells more resistant to 5-FU via PTEN/PI3K/ AKT signaling pathway (Figure 6). Considering that the experiments were only carried out in vitro, we believe the next step should involve in vivo experiments in animals and performed flow cytometry to detect cell apoptosis and cycle. Further studies will focus on whether miR-499a-5p could be used as a potential biomarker for patient risk stratification and local regional metastasis in PC.

\section{Acknowledgments}

Funding: None.

\section{Footnote}

Reporting Checklist: The authors have completed the MDAR reporting checklist. Available at https://dx.doi. org/10.21037/atm-21-6556

Data Sharing Statement: Available at https://dx.doi. org/10.21037/atm-21-6556 
Conflicts of Interest: All authors have completed the ICMJE uniform disclosure form (available at https://dx.doi. org/10.21037/atm-21-6556). The authors have no conflicts of interest to declare.

Ethical Statement: The authors are accountable for all aspects of the work in ensuring that questions related to the accuracy or integrity of any part of the work are appropriately investigated and resolved. All procedures performed in this study involving human participants were in accordance with the Declaration of Helsinki (as revised in 2013). The present study obtained the approval of the Ethics Committee (No. CHEC-Y2020-043) of Changhai Hospital (Shanghai, China) and written informed consent was obtained from all participants.

Open Access Statement: This is an Open Access article distributed in accordance with the Creative Commons Attribution-NonCommercial-NoDerivs 4.0 International License (CC BY-NC-ND 4.0), which permits the noncommercial replication and distribution of the article with the strict proviso that no changes or edits are made and the original work is properly cited (including links to both the formal publication through the relevant DOI and the license). See: https://creativecommons.org/licenses/by-nc-nd/4.0/.

\section{References}

1. Siegel RL, Miller KD, Jemal A. Cancer statistics, 2019. CA Cancer J Clin 2019;69:7-34.

2. Ferlay J, Shin HR, Bray F, et al. Estimates of worldwide burden of cancer in 2008: GLOBOCAN 2008. Int J Cancer 2010;127:2893-917.

3. Zhai S, Huo Z, Wang Y, et al. TRIANGLE operation for borderline resectable pancreatic cancer in total pancreatectomy. Transl Cancer Res 2019;8:2416-24.

4. Yu H, Gao G, Jiang L, et al. Decreased expression of miR218 is associated with poor prognosis in patients with colorectal cancer. Int J Clin Exp Pathol 2013;6:2904-11.

5. Moertel CG. Chemotherapy for pancreatic cancer. N Engl J Med 1994; 330:1136-42.

6. Poon MA, O'Connell MJ, Moertel CG, et al. Biochemical modulation of fluorouracil: evidence of significant improvement of survival and quality of life in patients with advanced colorectal carcinoma. J Clin Oncol 1989;7:1407-18.

7. Ebert MS, Sharp PA. Roles for microRNAs in conferring robustness to biological processes. Cell 2012;149:515-24.
8. Saliminejad K, Khorram Khorshid HR, Soleymani Fard $\mathrm{S}$, et al. An overview of microRNAs: Biology, functions, therapeutics, and analysis methods. J Cell Physiol 2019;234:5451-65.

9. Yan Y, Wang R, Guan W, et al. Roles of microRNAs in cancer associated fibroblasts of gastric cancer. Pathol Res Pract 2017;213:730-6.

10. Acunzo M, Romano G, Wernicke D, et al. MicroRNA and cancer--a brief overview. Adv Biol Regul 2015;57:1-9.

11. Hayes J, Peruzzi PP, Lawler S. MicroRNAs in cancer: biomarkers, functions and therapy. Trends Mol Med 2014;20:460-9.

12. Zhao L, Wang B, Zhang W, et al. Effect of miR-499a$5 p$ on damage of cardiomyocyte induced by hypoxiareoxygenation via downregulating CD38 protein. J Cell Biochem 2020;121:996-1004.

13. He S, Li Z, Yu Y, et al. Exosomal miR-499a-5p promotes cell proliferation, migration and EMT via mTOR signaling pathway in lung adenocarcinoma. Exp Cell Res 2019;379:203-13.

14. Sun JC, Zheng B, Sun RX, et al. MiR-499a-5p suppresses apoptosis of human nucleus pulposus cells and degradation of their extracellular matrix by targeting SOX4. Biomed Pharmacother 2019;113:108652.

15. Neshati V, Mollazadeh S, Fazly Bazzaz BS, et al. MicroRNA-499a-5p Promotes Differentiation of Human Bone Marrow-Derived Mesenchymal Stem Cells to Cardiomyocytes. Appl Biochem Biotechnol 2018;186:245-55.

16. Liu J, Huang L, Su P, et al. MicroRNA-499a-5p inhibits osteosarcoma cell proliferation and differentiation by targeting protein phosphatase 1D through protein kinase $\mathrm{B} / \mathrm{glycogen}$ synthase kinase $3 \beta$ signaling. Oncol Lett 2018;15:4113-20.

17. Liu X, Zhang Z, Sun L, et al. MicroRNA-499-5p promotes cellular invasion and tumor metastasis in colorectal cancer by targeting FOXO4 and PDCD4. Carcinogenesis 2011;32:1798-805.

18. Schmittgen TD, Livak KJ. Analyzing real-time PCR data by the comparative $\mathrm{C}(\mathrm{T})$ method. Nat Protoc 2008;3:1101-8.

19. Du W, Zhang X, Wan Z. miR-3691-5p promotes hepatocellular carcinoma cell migration and invasion through activating PI3K/Akt signaling by targeting PTEN. Onco Targets Ther 2019;12:4897-906.

20. McQuade RM, Stojanovska V, Bornstein JC, et al. Colorectal Cancer Chemotherapy: The Evolution of Treatment and New Approaches. Curr Med Chem 
2017;24:1537-57.

21. Zhang Y, Talmon G, Wang J. MicroRNA-587 antagonizes 5-FU-induced apoptosis and confers drug resistance by regulating PPP2R1B expression in colorectal cancer. Cell Death Dis 2016;7:e2525.

22. Hua L, Zhu G, Wei J. MicroRNA-1 overexpression increases chemosensitivity of non-small cell lung cancer cells by inhibiting autophagy related 3-mediated autophagy. Cell Biol Int 2018;42:1240-9.

23. Zhang Y, Hu X, Miao X, et al. MicroRNA-425-5p regulates chemoresistance in colorectal cancer cells via regulation of Programmed Cell Death 10. J Cell Mol Med 2016;20:360-9.

24. Di Cristofano A, Pandolfi PP. The multiple roles of PTEN in tumor suppression. Cell 2000;100:387-90.

25. Li Y, Vecchiarelli-Federico LM, Li YJ, et al. The miR-1792 cluster expands multipotent hematopoietic progenitors whereas imbalanced expression of its individual oncogenic miRNAs promotes leukemia in mice. Blood 2012;119:4486-98.

26. Pasqualini L, Bu H, Puhr M, et al. miR-22 and miR29a Are Members of the Androgen Receptor Cistrome Modulating LAMC1 and Mcl-1 in Prostate Cancer. Mol Endocrinol 2015;29:1037-54.

27. Huse JT, Brennan C, Hambardzumyan D, et al. The PTEN-regulating microRNA miR-26a is amplified in high-grade glioma and facilitates gliomagenesis in vivo. Genes Dev 2009;23:1327-37.

Cite this article as: Ouyang L, Liu RD, Lei DQ, Shang QC, Li HF, Hu XG, Zheng H, Jin G. MiR-499a-5p promotes 5 -FU resistance and the cell proliferation and migration through activating PI3K/Akt signaling by targeting PTEN in pancreatic cancer. Ann Transl Med 2021;9(24):1798. doi: 10.21037/atm-21-6556
28. Meng F, Henson R, Wehbe-Janek H, et al. MicroRNA-21 regulates expression of the PTEN tumor suppressor gene in human hepatocellular cancer. Gastroenterology 2007;133:647-58.

29. Filbin MG, Dabral SK, Pazyra-Murphy MF, et al. Coordinate activation of Shh and PI3K signaling in PTEN-deficient glioblastoma: new therapeutic opportunities. Nat Med 2013;19:1518-23.

30. Dey A, Wong E, Kua N, et al. Hexamethylene bisacetamide (HMBA) simultaneously targets AK'T and MAPK pathway and represses NF kappaB activity: implications for cancer therapy. Cell Cycle 2008;7:3759-67.

31. Yang J, Song Q, Cai Y, et al. RLIP76-dependent suppression of PI3K/AKT/Bcl-2 pathway by miR-101 induces apoptosis in prostate cancer. Biochem Biophys Res Commun 2015;463:900-6.

32. Matsunaga T, Takemoto N, Sato T, et al. Interaction between leukemic-cell VLA-4 and stromal fibronectin is a decisive factor for minimal residual disease of acute myelogenous leukemia. Nat Med 2003;9:1158-65.

33. Huang M, Tang SN, Upadhyay G, et al. Embelin suppresses growth of human pancreatic cancer xenografts, and pancreatic cancer cells isolated from KrasG12D mice by inhibiting Akt and Sonic hedgehog pathways. PLoS One 2014;9:e92161.

(English Language Editor: J. Gray) 